\title{
Automultiscopic displays based on orbital angular momentum of light
}

This content has been downloaded from IOPscience. Please scroll down to see the full text.

2016 J. Opt. 18085608

(http://iopscience.iop.org/2040-8986/18/8/085608)

View the table of contents for this issue, or go to the journal homepage for more

Download details:

IP Address: 131.111.184.102

This content was downloaded on 03/05/2017 at 09:34

Please note that terms and conditions apply.

You may also be interested in:

Roadmap on structured light

Halina Rubinsztein-Dunlop, Andrew Forbes, M V Berry et al.

Simulating thick atmospheric turbulence in the lab with application to orbital angular momentum communication

Brandon Rodenburg, Mohammad Mirhosseini, Mehul Malik et al.

Measurement of the light orbital angular momentum spectrum using an optical geometrictransformation

Martin P J Lavery, Gregorius C G Berkhout, Johannes Courtial et al.

Design challenges and guidelines for free-space optical communication links using

orbital-angular-momentum multiplexing of multiple beams

Alan E Willner, Guodong Xie, Long Li et al.

Efficient sorting of free electron orbital angular momentum

Benjamin J McMorran, Tyler R Harvey and Martin P J Lavery

Propagation of orbital angular momentum carrying beams through a perturbing medium

Abraham Chaibi, Cosmas Mafusire and Andrew Forbes

Free space propagation of concentric vortices through underwater turbid environments

K S Morgan, J K Miller, B M Cochenour et al.

Quantum state tomography of orbital angular momentum photonic qubits via a projection-based technique

Adrien Nicolas, Lucile Veissier, Elisabeth Giacobino et al. 


\title{
Automultiscopic displays based on orbital angular momentum of light
}

\author{
Xuefeng $\mathrm{Li}^{1}$, Jiaqi $\mathrm{Chu}^{1}$, Quinn Smithwick ${ }^{2}$ and Daping $\mathrm{Chu}^{1}$ \\ ${ }^{1}$ Photonics and Sensors Group, Department of Engineering, University of Cambridge, UK \\ ${ }^{2}$ Disney Research, Glendale, CA, USA \\ E-mail: dpc31@cam.ac.uk
}

Received 28 January 2016, revised 14 March 2016

Accepted for publication 5 April 2016

Published 20 July 2016

\begin{abstract}
Orbital angular momentum (OAM) of light has drawn increasing attention due to its intriguingly rich physics and potential for a variety of applications. Having an unbounded set of orthogonal states, OAM has been used to enhance the channel capacity of data transmission. We propose and demonstrate the viability of using OAM to create an automultiscopic 3D display. Multi-view image information is encoded using an OAM beam array, then sorted into different view directions using coordinate transformation elements. A three-view demonstration was achieved to encode and decode $9 \times 9$ pixel images. These demonstrations suggest that OAM could potentially serve as an additional platform for future 3D display systems.
\end{abstract}

Keywords: orbital angular momentum, display, automultiscopic 3D

(Some figures may appear in colour only in the online journal)

\section{Introduction}

The recent convergence of advances in display technology, image capturing, signal processing and optical communications has generated a paradigm shift in how information is collected, conveyed and rendered. This area of research has been developed rapidly due to the widespread use of flat panel displays [1] and spatial light modulators (SLMs), especially those based on liquid crystals (LC) [2]. In particular, the production of and consumer interest in 3D content and technologies, such as virtual reality and augmented reality, have been increasing exponentially in the past few years. 3D display technology combines both rendering and conveyance of spatial information [3-5]. The state-of-the-art autostereoscopic 3D display technologies can be classified into three broad categories: (1) multiview 3D display [6], (2) volumetric 3D display [7], and (3) digital holographic display [8]. Among existing multiview encoded 3D display approaches, there are various mechanisms including [4]: occlusion-based technologies (parallax barrier, time-sequential aperture, moving slit, and cylindrical parallax barrier), refraction-based (lenticular sheet, multiprojector, and integral imaging), reflection-based, diffraction-based, and eye tracking. One of the most significant drawbacks in above methods is the tradeoff between image resolution in each view and the number of viewpoints [9]. Although using a display panel with smaller pixel size $(\sim 5 \mu \mathrm{m})$ could potentially increase the viewing angle while keeping the display resolution, the achievable number of views is limited thus compromising smooth parallax viewing. In addition to multiview approaches, a number of binocular (two-view) stereoscopic 3D display techniques have already been commercialized due to their high quality and low cost. Nevertheless, the need to wear polarization or shutter glasses is a major concern; polarization supports only two-views thus supporting only a single correct perspective and requiring tracking for varying viewpoints; while shutter glasses require active glasses.

Nevertheless, advancements in fundamental science may pave the way for 3D displays with totally different technologies. As we know, the wavefront completely characterizes the radiance flowing through all the points in a plane, in all possible directions, and of all possible curvatures. The ultimate goal of 3D display systems is to faithfully reproduce the wavefront, but it is almost impossible to practically realize due to its huge information requirements. Instead, a subsampling of views is implemented to mimic the 3D information. Different views are encoded and multiplexed in various means, then separated when the information is received by the observer. The use of multiplexing and demultiplexing in free space and optical fiber 


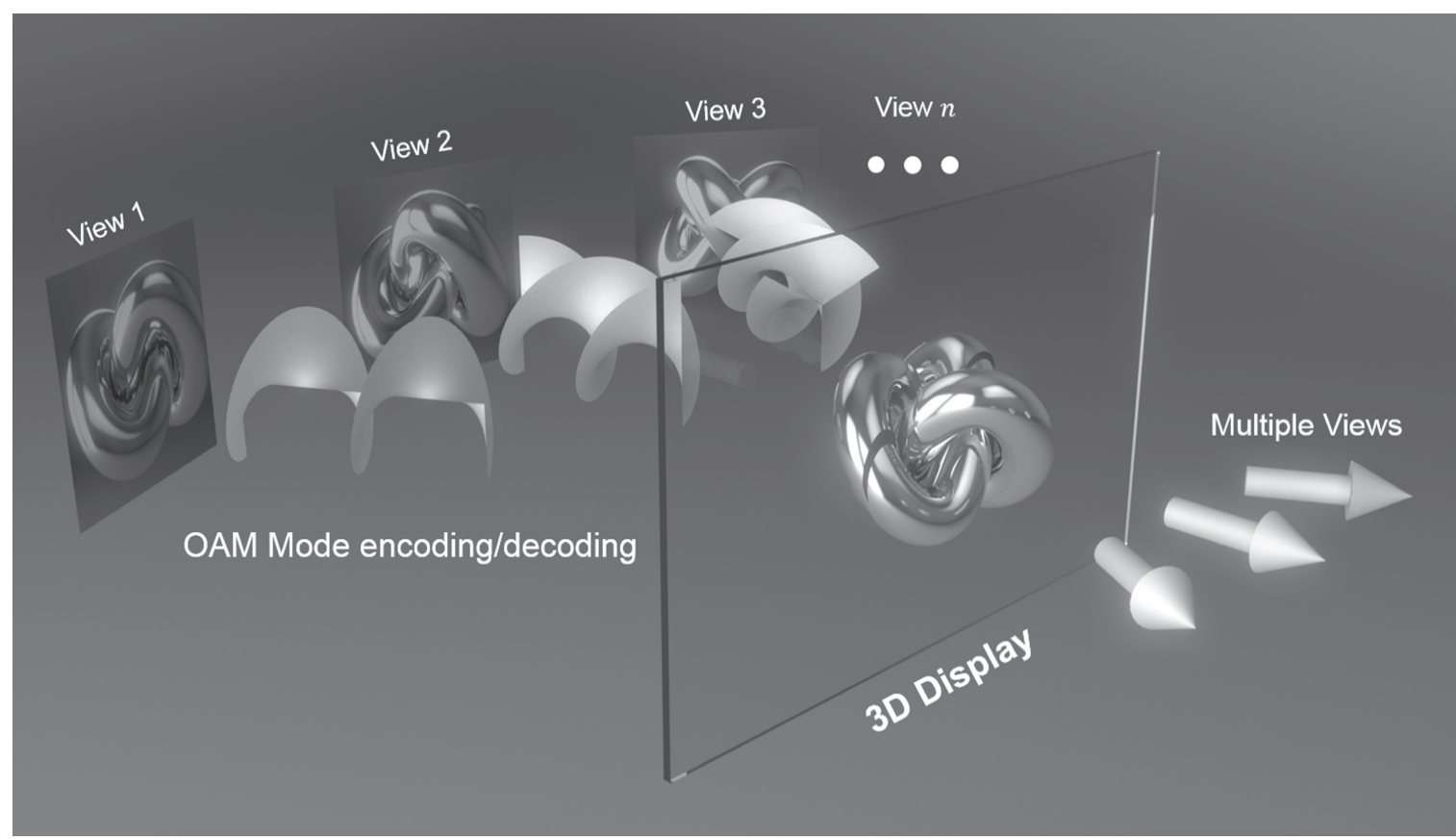

Figure 1. Schematic illustration of a multiple-view 3D display using orbital angular momentum of light.

communications has benefited from the encoding/decoding using light's various degrees of freedom [10, 11]. Among them include wavelength/frequency, amplitude/phase, time sequence, and polarization of light, each of which can be solely or jointly implemented to achieve high data-carrying capacity. Similarly, the development of multiview 3D display techniques has been focused on separating view channels with respect to independent variables, e.g. time multiplexing (time) [12] and integral imaging (space) [13].

Angular momentum is one of the most fundamental physical quantities in both classical and quantum physics [14]. It has long been known that the circular polarization state of electromagnetic waves (e.g. visible light) is associated with spin angular momentum [15]. In contrast, the orbital component of angular momentum in optics is affiliated with a vortex-like phase profile $\mathrm{e}^{\mathrm{i} l \theta}[16]$, where $l$ is the topological charge, and $\theta$ is the azimuthal angle. Optical beams with this helical phase have been shown to possess a well-defined orbital angular momentum (OAM) of $l \hbar$ per photon. In recent decades, OAM of light has drawn tremendous attention [17] due to its potential application in communication [18, 19], imaging [20], optical tweezers [21], etc. In particular, OAM is very attractive for data transmission due to its nature of unlimited number of potential states so that OAM beams can carry an infinite amount of data in principle. It has been demonstrated that free-space and optical fiber communication with OAM multiplexing/demultiplexing can work at broadband frequency with much higher data rate capacity [18, 19]. There are two approaches to increase the channel capacity with OAM, either by encoding information as OAM states of the beam or by using OAM beams as information carriers for multiplexing. In the field of 3D display, if we can encode each view with one of the OAM eigen-state, a 3D display with unlimited views can theoretically be constructed, as illustrated in figure 1 .

In this paper, we present an autostereoscopic multi-view 3D display architecture based on the multiplexing and demultiplexing of OAM-carrying beams. First, we describe the concept of encoding of 2D image information using OAM modes, multiplexing of different OAM channels, then simultaneous separation of different images into various viewing angles. Experimentally, a three-view proof-of-concept display is demonstrated, where three $9 \times 9$ pixel images are encoded and spatial separated using OAM beam arrays. Our approach efficiently breaks the dependence between number of available views and the image resolution. Moreover, the compatibility between OAM based 3D display and data communication may provide the possibility for all-optical collection, transmission, and rendering of 3D information. This might pave the way for the next generation of multiview 3D display technologies.

\section{Concept and optics design}

For data transmission both in free space or optical fiber communications, OAM modes serve as a carrier of information and they are regarded as an additional degree of freedom which is complementary to the existing multiplexing technologies, permitting a greatly increased data-carrying capacity [19]. However, the information is generally encoded time sequentially as a bit-stream for each OAM channel. Thus a 2D static image is typically transmitted pixel by pixel [22] in a raster order, similar to the reciprocal way of a CRT display. Generally, a 2D image can be expressed as a two-dimensional function associated with a complex field amplitude. In conventional imaging system, this $2 \mathrm{D}$ information is imprinted 


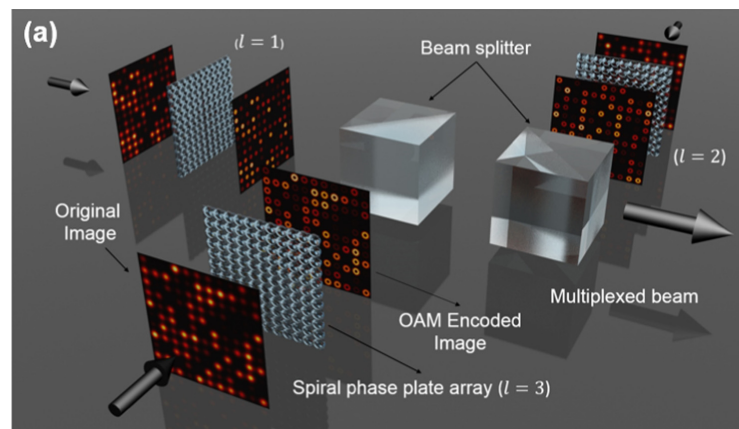

(b)

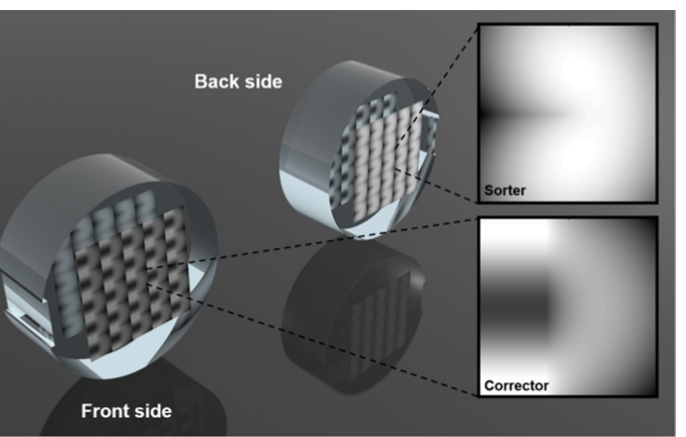

Figure 2. (a) Conceptual illustration of multiplexing images with three OAM states. (b) Designed sorter and corrector array for sorting of OAM beam lattice. The height profiles of the sorter and corrector are shown in the insets, respectively.

onto the optical beam using an SLM. Due to the lack of complex phase structures in zero-order Hermite-Gaussian beams, the encoded images can be relayed without much distortion. However, direct superposition of the complex field of an image onto an OAM helical phase structure would lead to the breakdown of OAM eigen-modes in the far field and therefore large crosstalk between different channels. Nevertheless, there are another two mechanisms to incorporate the 2D information into OAM modes without disrupting the orthogonality between distinct states. (1) Convolution between an image and an OAM mode, which has been investigated extensively in spiral phase contrast imaging/ microscopy [23]; (2) encoding the 2D amplitude distribution as an array of OAM beams, where each OAM element corresponds to one pixel of the encoded image. In this paper we employ the array approach due to the fact that the convolution method requires complex optics in order to separate different views, while the latter one can benefit from the OAM mode sorting using coordinate transformation components. Once a number of images are encoded with different OAM modes, they can be multiplexed into one optical beam bundle using beam splitters, as shown in figure 2(a). Unlike their use in communication systems, multiplexed OAM beams would propagate no more than $5 \mathrm{~m}$ before the information is decoded by an OAM sorter and projected to the imaging plane. Thus the effect of air turbulence can be neglected in this case [24].

To achieve the separation between different views, the coordinate transformation, which maps the azimuthal component $(0-2 \pi)$ into the Cartesian coordinates, can effectively unwrap a donut-like OAM beam into a rectangular shape with a tilted wavefront, as proposed and demonstrated by Padgett's group [25]. The OAM sorter performs a mapping from the input plane $(x, y)$ to the output plane $(u, v)$, which can be described as: $\quad v=a \arctan (y / x) \quad$ and $u=-a \ln \left(\sqrt{x^{2}+y^{2} / b}\right)$. And the phase profile of the transforming optical element is expressed as:

$$
\begin{aligned}
\phi_{1}(x, y) & =\frac{2 \pi a}{\lambda f} \\
\times & {\left[y \arctan \left(\frac{y}{x}\right)-x \ln \left(\frac{\sqrt{x^{2}+y^{2}}}{b}\right)+x\right] }
\end{aligned}
$$

and the phase aberrations caused by the transformation can be corrected by a second element as:

$$
\phi_{2}(u, v)=-\frac{2 \pi a}{\lambda f} \exp \left(-\frac{u}{a}\right) \cos \left(\frac{v}{a}\right)
$$

where $a=d / 2 \pi$ scales the size of the transformed beam and $b$ determines the position of the output beam. $\lambda$ is the wavelength of the light, and $f$ is the focal length of the lens between the above two elements. By constructing an array of sorter and corrector pairs, we can effectively route different OAM beam arrays into different directions and generate a multiview 3D image.

The designed phase profile can be generated using standard phase-only SLMs, but diffraction based SLMs have limited diffraction efficiency and the pixelated nature of SLM (pitch $\sim 10 \mu \mathrm{m}$ ) would result in poor performance of OAM sorting [25]. Instead we use refractive elements to carry out the sorting process [26] and the surface profile of the sorter can be arbitrarily manufactured using diamond machining. In addition, the Fourier transform lens can be incorporated into the first element and corrected on the second element by another lens term. By carefully tailoring the parameters, the designed freeform surfaces can be fabricated on the front and back sides of a single optical plate respectively, which allows for compactness and precise alignment of optics, as shown in figure 2(b).

\section{Simulation}

First, we consider the generation of OAM beam arrays. A variety of ways have been implemented to generate single OAM beam with helical phase profile, such as spiral phase plate (SPP) [27], q-plate [28], combination of HermiteGaussian modes [16], and SLMs [29]. A computer-generated hologram can be used to transform the pseudo plane-wave laser beam into one with exotic phase structure. In this paper, we use the SPP to generate Laguerre-Gaussian (LG) beams as OAM-carrying states with different indices $l$. And ABCD matrix method [30] is applied to simulate the propagation and wavefront shaping of optical waves. Figure 3(a) displays the intensity of the simulated LG beam when a Gaussian beam with the waist size $\left(w_{0}=1.0 \mathrm{~mm}\right)$ passes through a SPP with 

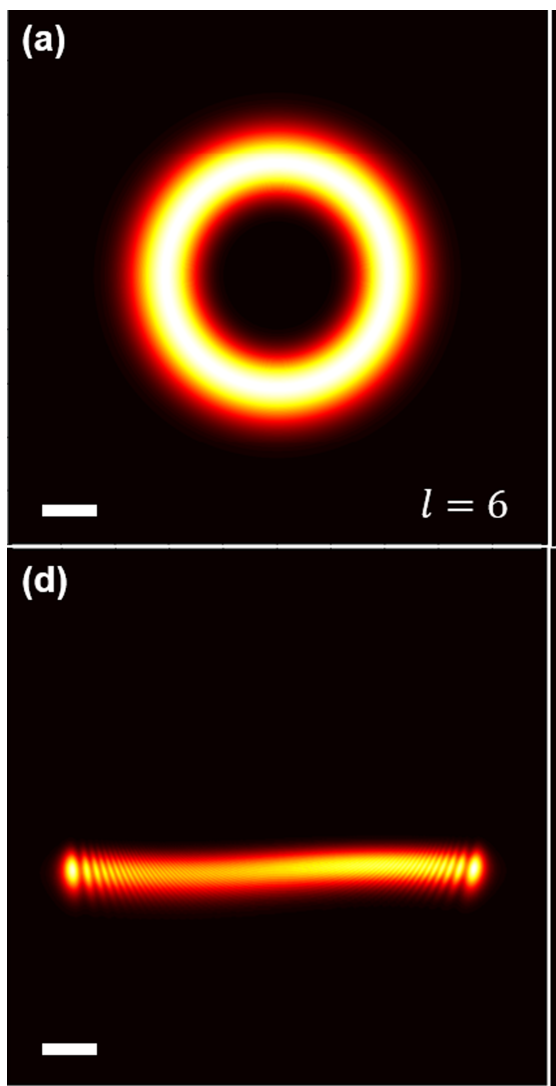

(b)

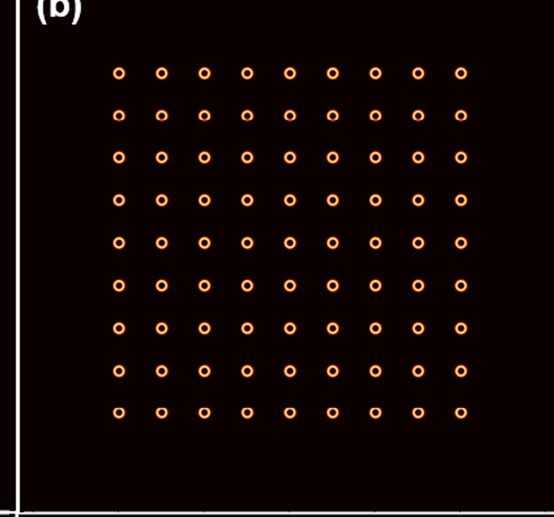

(e)

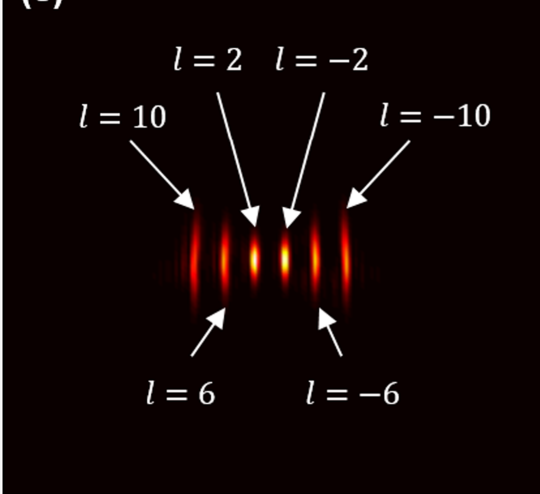

(c)
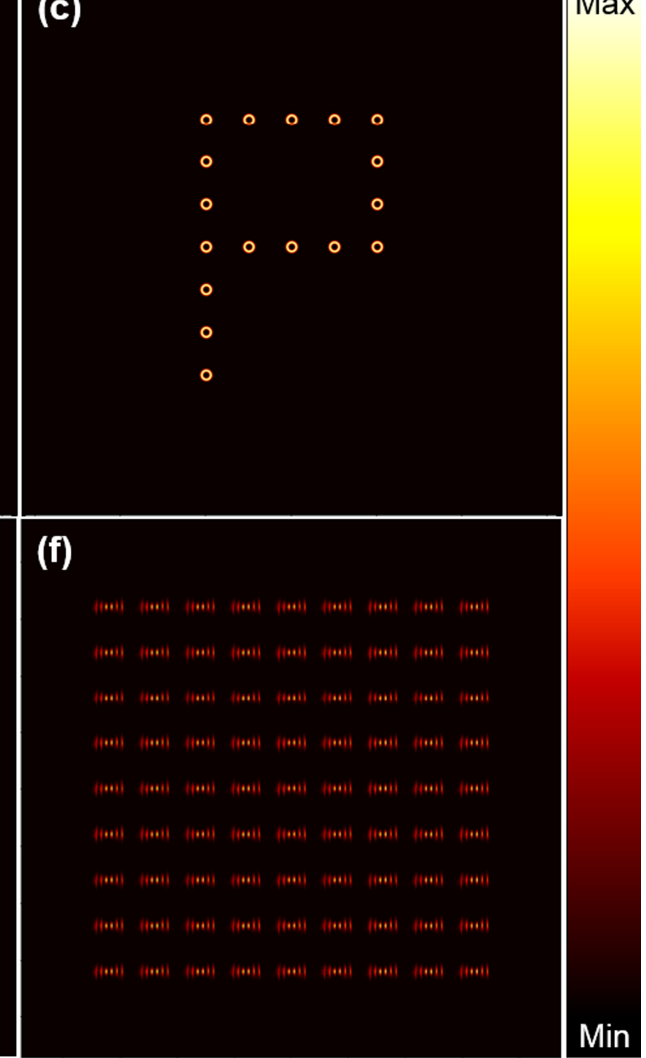

Figure 3. (a) Simulated intensity profile of a LG beam with index $l=6$. (b) The modeled intensity of a $9 \times 9$ array of OAM-carrying LG beams generated by beam copying device. (c) Transmitted image of the letter ' $\mathrm{P}$ ' after passing through an image mask. (d) Intensity profile of the coordinate transformed OAM beam $(l=6)$ directly after the corrector. (e) and (f) Simulated OAM spectra of (e) a single and (f) an array of superposed LG modes with $l=-10,-6,-2,2,6,10$. The scale of the white bar is $1.0 \mathrm{~mm}$.

a charge $(l=6)$. Inspired by Mirhosseini et al [31], we investigate the transformation of a single OAM beam to a square lattice of optical vortices using the beam copying device. As shown in figure 3(b), we use a phase-only hologram to make multiple coherent copies $(9 \times 9)$ of the LG beams with identical amplitude and phase profiles. The phase structure of this element can be written as:

$$
\begin{aligned}
\Psi= & \tan ^{-1}\left(\frac{\sum_{m=-M}^{M} \gamma_{m} \sin \left[(2 \pi s / \lambda) m x+\alpha_{m}\right]}{\sum_{m=-M}^{M} \gamma_{m} \cos \left[(2 \pi s / \lambda) m x+\alpha_{m}\right]}\right) \\
& +\tan ^{-1}\left(\frac{\sum_{n=-N}^{N} \gamma_{n} \sin \left[(2 \pi s / \lambda) n y+\alpha_{n}\right]}{\sum_{n=-N}^{N} \gamma_{n} \cos \left[(2 \pi s / \lambda) n y+\alpha_{n}\right]}\right),
\end{aligned}
$$

where $2 M+1$, and $2 N+1$ are the number of copies of the OAM beam in horizontal and vertical directions, respectively. $s$ is the angular separation between adjacent copy, while $\gamma_{m, n}$ and $\alpha_{m, n}$ are relative amplitude and phase parameters corresponding to separate diffraction orders. These parameters are optimized to achieve uniformity between the multiple copies. Then light propagating through the diffraction beam splitter and a corrector is collected by an aligned lenslet array that directs the OAM beam array onto an image mask. The mask is also aligned with the beam array and produces the desired OAM-encoded view image. Figure 3(c) shows the transmitted image of the letter ' $\mathrm{P}$ ' consisting of OAM pixels.

The sorter converts the azimuthal phase gradient into tilted plane waves (i.e. linear phase gradient) with the tilt of a resulting plane wave proportional to the pitch of a spiral wavefront $\mathrm{e}^{\mathrm{i} l x}$. The phase correction plate removes residual phase aberrations caused by the sorter element. Additional fan-out and phase correctors can be used to reduce the crosstalk between OAM modes. Figure 3(d) shows the intensity of a coordinate transformed OAM beam $(l=6)$ just after the phase corrector. Note, the transformed optical beam is not a perfect rectangular shape, but has a sinusoidal distortion due to the nonzero skew angle of OAM beams [25]. The parameters of the sorter are: $d=8 \mathrm{~mm}$, and $b=0.004$. Passing the tilted wavefronts through a lens, the lateral position $\left(t_{1}\right)$ of each focused beam varies with the tilt of the incoming plane wave. Hence the propagation direction of different transformed OAM beams can be expressed as: $\tan \theta=\frac{t_{1}}{f}=\frac{\lambda l}{d}$, where $\theta$ is the divergence angle. Figure 3(e) shows the modeled intensity profile of the sorting of six equally superposed OAM modes on the image plane, while the index separation between adjacent modes is $\Delta l=4$. Although, these modes are well separated with each other on the imaging plane, the angular separation $\left(\Delta \theta=0.02^{\circ}\right.$ for $d=8 \mathrm{~mm}$ ) is still too small compared with a conventional multiview 3D display $\left(\Delta \theta>0.7^{\circ}\right)$. Therefore, both the size 

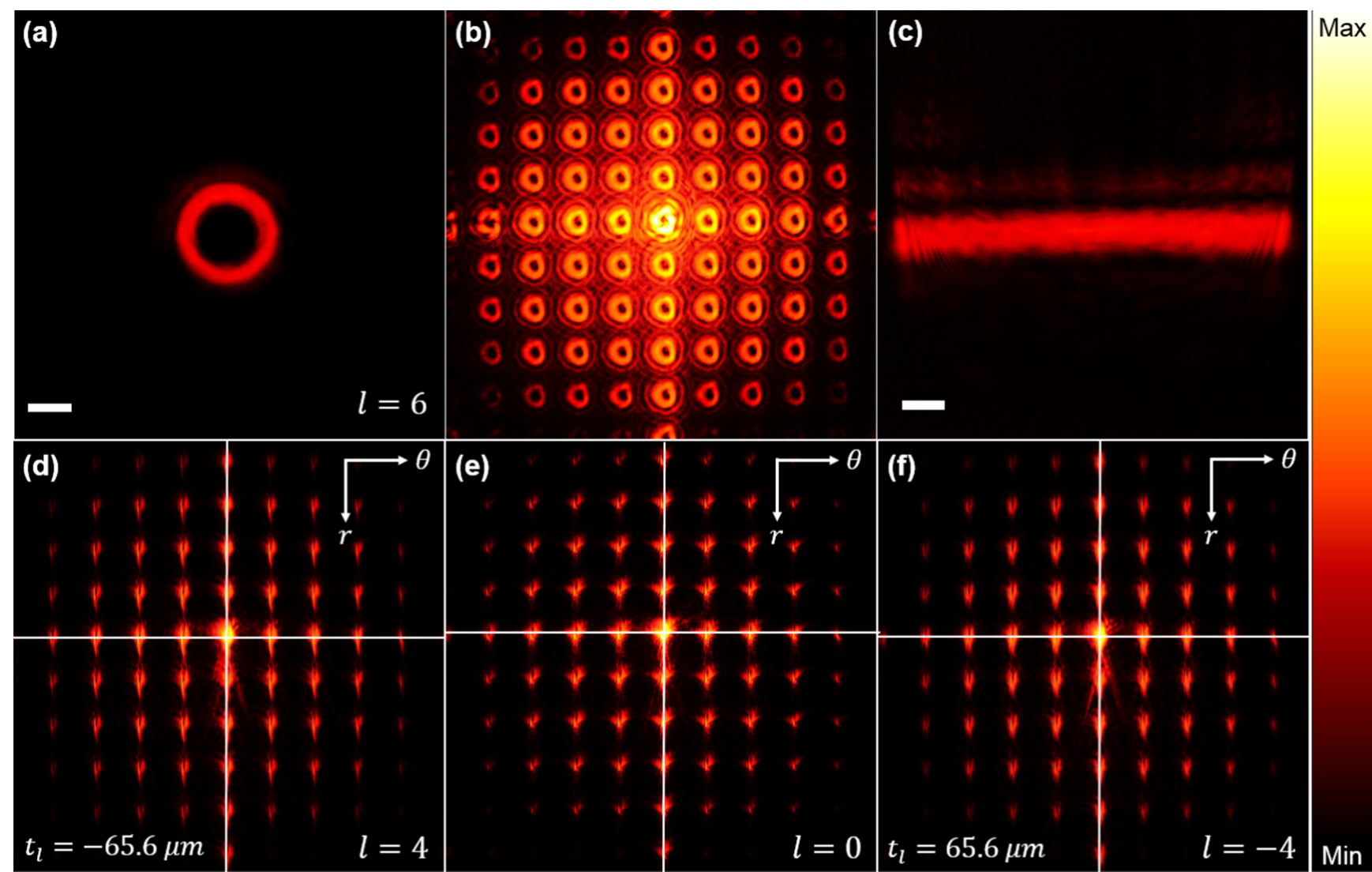

Figure 4. (a) Measured intensity profile of a LG beam with an index $l=6$. (b) The observed intensity of a $9 \times 9$ array of OAM-carrying LG beams generated by Dammann grating. (c) Measured intensity profile of the coordinate transformed OAM beam directly after the corrector. (d)-(f) Experimentally measured OAM spectra of an array of different LG modes with (d) $l=4$, (e) $l=0$, and (f) $l=-4$. The scale of the white bar is $2.0 \mathrm{~mm}$.

of OAM beam and sorter parameter should be reduced to enable a noticeable viewing angle separation. Figure 3(f) shows the intensity profile of the sorted OAM array constituted of equally weighted LG beams with the scaling factor $d=1 \mathrm{~mm}$ on each sorter pixel. If each OAM array is coded with a different image mask, we are able to construct a six view $3 \mathrm{D}$ display using the sorter array.

\section{Experiment}

In the experiment, we generated the input OAM test modes by use of a SPP, which was manufactured using laser direct writing method by RPC Photonics. The SPP has equally spaced sub-areas $(10 \mathrm{~mm} \times 10 \mathrm{~mm})$ with an adjustable helical index $l$ ranging from -8 to 8 . The Gaussian beam emitted from a $632.8 \mathrm{~nm}$ HeNe laser was expanded by a pair of lenses $\left(f_{1}=10 \mathrm{~mm}, f_{2}=100 \mathrm{~mm}\right)$ and illuminated onto the SPP, producing the desired OAM state sequentially. Two adjustable apertures were placed in front and at the back of the SPP respectively to remove the noise outside the area of the expanded Gaussian and LG beam. Figure 4(a) shows a measured far-field intensity pattern of an OAM-carrying beam with a charge $l=6$. In order to produce an OAM beam array with equally distributed energy and high diffraction efficiency, we encoded a Dammann grating structure [32] onto a
LC on silicon SLM (256 gray level, $15 \mu \mathrm{m}$ pixel pitch). The advantage of using the Dammann grating instead of fan-out component is that the beam copying process discussed above requires both transformation and correction [31], which adds complexity into the optics alignment. In addition, the phase structure of a Dammann grating is binary, allowing for cost effective fabrication using standard photo-lithography and easy integration to other systems. The phase values on a Dammann grating, either 0 or $\pi$, are encoded on a series of transition points within each period. The positions of these transition points are optimized to achieve maximum diffraction efficiency. We designed the Dammann grating (period: $150 \mu \mathrm{m})$ according to the transition points listed in [33] achieving a $9 \times 9$ array of OAM carrying beams, as shown in figure 4(b). The observed OAM array exhibits good quality period and intensity distributions. The Airy ring pattern surrounding each OAM pixel was due to the use of circular apertures in our set up.

The OAM sorter and corrector were designed to increase the measurement bandwidth by use of a large aperture $(d=18 \mathrm{~mm})$ and small element distance $\left(D_{1,2}=300 \mathrm{~mm}\right)$. The two-part OAM sorting components were fabricated using a diamond machining method by Precision Optics Laboratory in Durham University. A Nanotech three-axis ultra-precision lathe was used in combination with a Nanotech NFTS6000 fast tool servo system to provide a fast axis superimposed on 
one of the axes of the lathe. The detailed fabrication process can be found at [34]. The generated OAM beam propagated through the elements, unwrapping the donut-like beam, and mapping the azimuthal coordinate into a transverse axis. Figure 4(c) shows the intensity of a coordinate transformed OAM beam with the charge $l=6$, exhibiting good rectangular shape.

In order to demonstrate the sorting performance of a uniformly distributed OAM lattice, we illuminated the sorted single OAM beam onto the Dammann grating hologram displayed on the SLM and a CCD camera was placed at the focal plane of the final focusing lens. The measured OAM spectra are shown in figures 4(d)-(f), which corresponds to OAM modes $l=4,0,-4$, respectively. The horizontal and vertical lines in these images mark the center of the zero order. The distortion to the desired elongated spot array arises from the misalignment of the optics. A shift in the transverse direction can be observed with a distance of $65.6 \mu \mathrm{m}$ separating the adjacent OAM modes. This measured shift is consistent with the theoretical model $t_{1}=\frac{\lambda l}{d} f$, which gives a lateral displacement of $17.6 \mu \mathrm{m}$ for an increase of OAM mode difference $\Delta l=1$. We observed a shift of pattern in the vertical direction due to the size of the OAM beam expanding with the increase of mode number. For example, the intensity of a normal zero order Hermite-Gaussian beam has its maximum at the beam center, while the intensity maximum of an LG beam lies on the ring. Therefore, we can observe a jump of spots centers in the original radial direction, as shown in figures 4(d)-(f).

After demonstrating the sorting performance of the OAM beam array, the designed image masks were inserted into the optics for 3D display purposes. As shown in figures 5(a)-(c), a group of three image masks were designed with each mask designated one of the letters ' $\mathrm{P}$ ', ' $S$ ', and ' $G$ '. These masks were fabricated on transparent films with the dark areas designed to block the incident light. Figures 5(d)-(f) show the observed image on the CCD when each letter is encoded with an OAM state $(l=7,0,-7)$ respectively. The shapes of the original images were well reconstructed in each case and they are consisted of elongated pixels. The noise in these images was due to the scattering on the surface of the inkjet printed masks. Most importantly, these three images were spatially separated due to the OAM mode difference. The transverse displacement in the horizontal axis is over $100 \mu \mathrm{m}$, which corresponds to a separation angle $0.015^{\circ}$.

\section{Discussion}

We have demonstrated a proof-of-concept three-view 3D display based on the mode orthogonality between OAMcarrying optical beams. The difference in OAM mode index provides a convenient way to sort multiple images by constructing a multiplexed OAM beam array. However, the angle between each views is very small $\left(0.015^{\circ}\right)$ compared to that of a conventional multiview $3 \mathrm{D}$ display $\left(>0.7^{\circ}\right)$ due to the large size of the sorter element parameter $(d=18 \mathrm{~mm})$. As discussed above, the separation angle can be written as $\theta=\arctan \frac{\lambda l}{d}$ and larger viewing angle can be achieved using sorter arrays with smaller scaling parameter $d$. Manufacturing of sorting elements with size $d<500 \mu \mathrm{m}$ is totally within the fabrication capabilities of our diamond machining equipment. Sub-micron resolution of this fast tool servo system can be obtained, which is sufficient for our optics design. Also, the working distance of this 3D display can be adjusted using relaying optics such as micro-lens array. Furthermore, the effective resolution of this 3D display system can be greatly improved with smaller pixel (OAM beam and sorter) size.

In this paper we study the angular separation between views, which is the basic requirement of a multiview 3D display. In addition, the coordinate transformation can be engineered to provide depth information of 3D scenes. The conformal mapping described in this study explores the polar to Cartesian transformation, which unwraps the phase gradient in the azimuthal direction into a transverse one. A reciprocal process can be implemented in order to transform the phase gradient from transverse direction to the radial direction (Cartesian to polar) [35]. If a lens is placed behind the transformed pattern, beams with different OAM index $l$ can be mapped to various depths along the beam axis. By combining both the angular tiling and depth mapping, a more comprehensive 3D display system can be constructed using images coded with OAM states.

Moreover, we have noticed that the radial component is also affected in the sorting process. This is because the beam waist enlarges with the increase of the OAM angular index $l$. If we are able to separately resolve the radial and angular components of an OAM-carrying beam, parallax viewing in both the horizontal and vertical directions is possible. To achieve this, Fourier transform of Bessel beams are chosen instead of SPP generated LG beams, which can be produced by programming the phase pattern on phase-only SLM [36]. The annular ring structure containing an azimuthal order can be arbitrarily engineered for simultaneous separation of OAM radial and azimuthal index by use of the sorter elements describe above.

In addition, the size of these pixels has no lower limit in principle. A large OAM capacity can be maintained by reducing the beam size (pixel size) and the element separation simultaneously. In practice, the pixel size here is usually determined by the resolution of the manufacturing process and the size of the display pixel can be manufactured in the tens of micrometers.

Finally, only the stereoscopic information is encoded and decoded here. However, the Cartesian-to-Polar coordinate transformation can be added to include the depth information by using two more elements. The view angle separation between adjacent modes is dependent on the element size and it increases with the reduction of the pixel size. Another way to increase the viewing angle separation is to reduce the number of OAM modes used in the 3D display. 

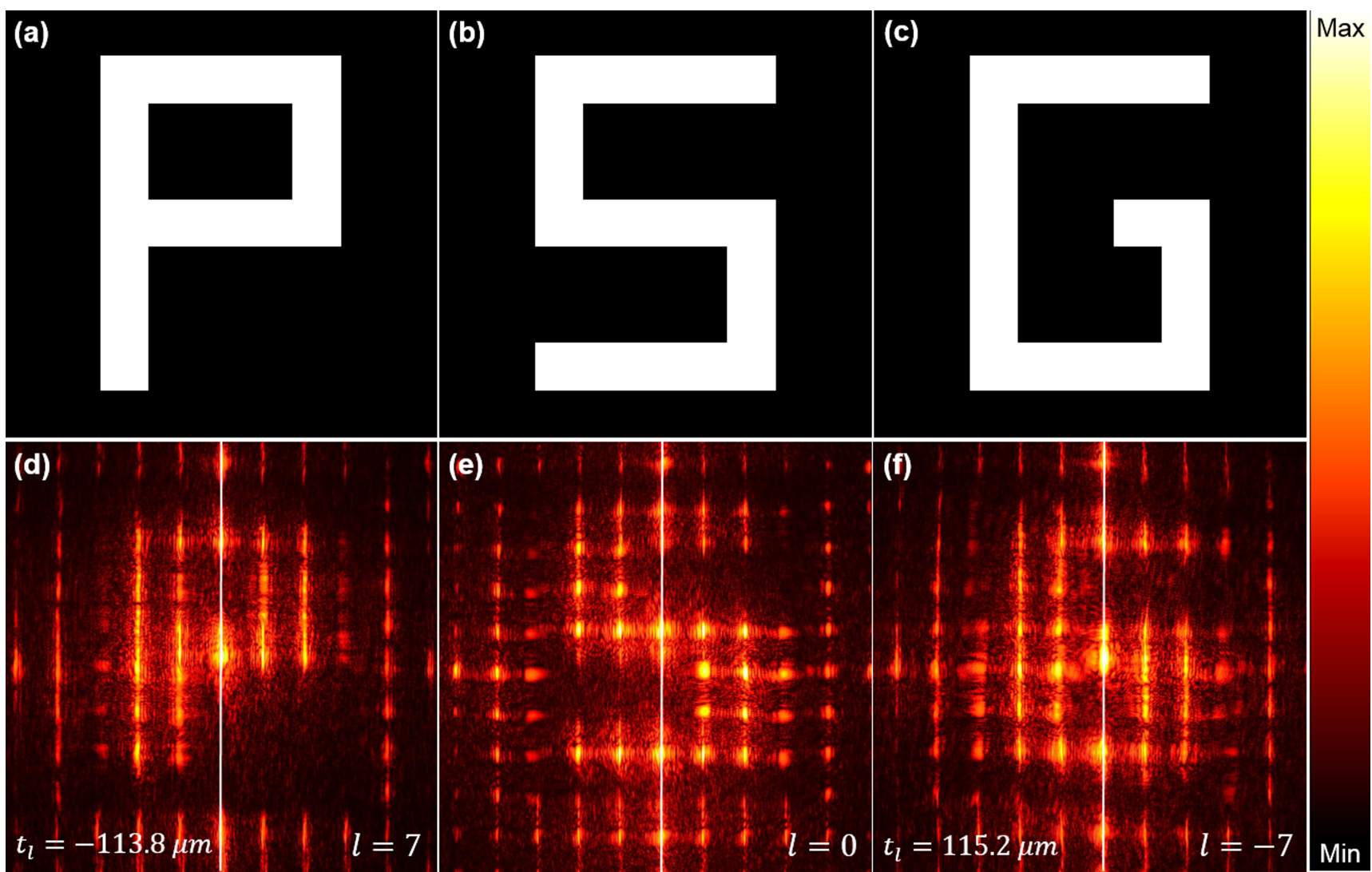

Figure 5. (a)-(c) Encoded image masks of letter 'P', 'S', and 'G', which are imprinted on transparent films. Each letter is associated with a distinct OAM mode with 'P' corresponds to OAM state $l=7$, 'S' to $l=0$, and 'G' to $l=-7$, respectively. (d)-(f) Observed image patterns on the CCD camera. The position of the measured image shifts on the horizontal axis, depending on the value of OAM state on which the image is encoded.

\section{Conclusion}

In conclusion, we have proposed and demonstrated, both theoretically and experimentally, a new multiview 3D display architecture using OAM based multiplexing and sorting. Information of different views are encoded into the OAM carrying beam array and decoded with a sorting element array. The phase gradient provided by OAM provides a genuine tool for sorting different modes and allows for multiview display. A three-view experiment was performed to demonstrate the pixel based 3D display, and sufficient to show capabilities beyond only two-views using polarization multiplexing. The separation angle between views can be further increased by using smaller sorter components. This design of autostereoscopic display may pave the way for future 3D display technologies.

\section{Acknowledgments}

This research was performed under a joint collaboration between Disney Research and the University of Cambridge through the CAPE consortium. XL, JC and DC would like to thank the UK Engineering and Physical Sciences Research Council (EPSRC) for the support through the Platform Grant for Liquid Crystal Photonics (EP/F00897X/1).

\section{References}

[1] Castellano J A 2012 Handbook of Display Technology (Amsterdam: Elsevier)

[2] Zhang Z, You Z and Chu D 2014 Fundamentals of phase-only liquid crystal on silicon (LCOS) devices Light: Sci. Appl. 3 e 21

[3] Dodgson N A 2005 Autostereoscopic 3D displays Computer 38 31-6

[4] Geng J 2013 Three-dimensional display technologies Adv. Opt. Photonics 5 456-535

[5] Holliman N S et al 2011 Three-dimensional displays: a review and applications analysis IEEE Trans. Broadcast. 57 $362-71$

[6] Son J-Y and Javidi B 2005 Three-dimensional imaging methods based on multiview images J. Disp. Technol. 1 $125-40$

[7] MacFarlane D L 1994 Volumetric three-dimensional display Appl. Opt. 33 7453-7

[8] Yoshikawa $\mathrm{H}$ and Yamaguchi T 2012 Recent progress on digital holography for 3D display SPIE/COS Photonics Asia (Beijing) (Optical Design and Testing V) paper 8557-11

[9] Hong J, Kim Y, Choi H-J, Hahn J, Park J-H, Kim H, Min S-W, Chen N and Lee B 2011 Three-dimensional display technologies of recent interest: principles, status, and issues [invited] Appl. Opt. $50 \mathrm{H} 87-115$

[10] Agrawal G P 2012 Fiber-Optic Communication Systems vol 222 (New York: Wiley)

[11] Richardson D J, Fini J M and Nelson L E 2013 Space-division multiplexing in optical fibres Nat. Photon. 7 354-62 
[12] Jang J-S and Javidi B 2003 Large depth-of-focus timemultiplexed three-dimensional integral imaging by use of lenslets with nonuniform focal lengths and aperturesizes Opt. Lett. 28 1924-6

[13] Xiao X, Javidi B, Martinez-Corral M and Stern A 2013 Advances in three-dimensional integral imaging: sensing, display, and applications [invited] Appl. Opt. 52 546-60

[14] Mandel L and Wolf E 1995 Optical Coherence and Quantum Optics (Cambridge: Cambridge University Press)

[15] Beth R A 1936 Mechanical detection and measurement of the angular momentum of light Phys. Rev. 50115

[16] Allen L, Beijersbergen M W, Spreeuw R J C and Woerdman J P 1992 Orbital angular momentum of light and the transformation of Laguerre-Gaussian laser modes Phys. Rev. A 458185

[17] Piccirillo B, Slussarenko S, Marrucci L and Santamato E 2013 The orbital angular momentum of light: genesis and evolution of the concept and of the associated photonic technology La Riv. Nuovo Cimento 36 501-54

[18] Wang J et al 2012 Terabit free-space data transmission employing orbital angular momentum multiplexing $\mathrm{Nat}$. Photon. 6 488-96

[19] Bozinovic N, Yue Y, Ren Y, Tur M, Kristensen P, Huang H, Willner A E and Ramachandran S 2013 Terabit-scale orbital angular momentum mode division multiplexing in fibers Science $3401545-8$

[20] Chen L, Lei J and Romero J 2014 Quantum digital spiral imaging Light: Sci. Appl. 3 e153

[21] O'Neil A T and Padgett M J 2000 Three-dimensional optical confinement of micron-sized metal particles and the decoupling of the spin and orbital angular momentum within an optical spanner Opt. Commun. 185 139-43

[22] Krenn M, Fickler R, Fink M, Handsteiner J, Malik M, Scheidl T, Ursin R and Zeilinger A 2014 Communication with spatially modulated light through turbulent air across Vienna New J. Phys. 16113028

[23] Torres J P and Torner L 2011 Twisted Photons: Applications of Light with Orbital Angular Momentum (New York: Wiley)

[24] Tyler G A and Boyd R W 2009 Influence of atmospheric turbulence on the propagation of quantum states of light carrying orbital angular momentum Opt. Lett. 34 $142-4$

[25] Berkhout G C G, Lavery M P J, Courtial J, Beijersbergen M W and Padgett M J 2010 Efficient sorting of orbital angular momentum states of light Phys. Rev. Lett. 105153601

[26] Lavery M P J, Robertson D J, Berkhout G C G, Love G D, Padgett M J and Courtial J 2012 Refractive elements for the measurement of the orbital angular momentum of a single photon Opt. Express 20 2110-5

[27] Sueda K, Miyaji G, Miyanaga N and Nakatsuka M 2004 Laguerre-Gaussian beam generated with a multilevel spiral phase plate for high intensity laser pulses Opt. Express 12 3548-53

[28] Marrucci L, Manzo C and Paparo D 2006 Optical spin-toorbital angular momentum conversion in inhomogeneous anisotropic media Phys. Rev. Lett. 96163905

[29] Mirhosseini M, Magana-Loaiza O S, Chen C, Rodenburg B, Malik M and Boyd R W 2013 Rapid generation of light beams carrying orbital angular momentum Opt. Express 21 30196-203

[30] Bernardo L M 1996 ABCD matrix formalism of fractional Fourier optics Opt. Eng. 35 732-40

[31] Mirhosseini M, Malik M, Shi Z and Boyd R W 2013 Efficient separation of the orbital angular momentum eigenstates of light Nat. Commun. 42781

[32] Krackhardt U and Streibl N 1989 Design of Dammann-gratings for array generation Opt. Commun. 74 31-6

[33] Dammann H and Klotz E 1977 Coherent optical generation and inspection of two-dimensional periodic structures $J$. Mod. Opt. 24 505-15

[34] Dow T A, Miller M H and Falter P J 1991 Application of a fast tool servo for diamond turning of nonrotationally symmetric surfaces Precis. Eng. 13 243-50

[35] Hossack W J, Darling A M and Dahdouh A 1987 Coordinate transformations with multiple computer-generated optical elements J. Mod. Opt. 34 1235-50

[36] Dudley A, Mhlanga T, Lavery M, McDonald A, Roux F S, Padgett M and Forbes A 2013 Efficient sorting of bessel beams Opt. Express 21 165-71 